\title{
Shifting to very early endoscopic DCR in acute suppurative dacryocystitis
}

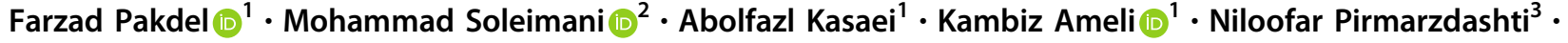 \\ Ali Sadeghi Tari ${ }^{1} \cdot$ Mehrbod Ghasempour $^{4} \cdot$ Ali Banafsheafshan ${ }^{4}$
}

Received: 6 March 2019 / Revised: 13 September 2019 / Accepted: 15 November 2019 / Published online: 10 December 2019

(c) The Author(s), under exclusive licence to The Royal College of Ophthalmologists 2019

\begin{abstract}
Purpose We aimed to show the outcome of very early endoscopic dacryocystorhinostomy (VE-EDCR) in a routine pool of patients with acute dacryocystitis (AD) and abscess formation compared with the standard late external dacryocystorhinostomy L-ExDCR.

Methods This was a prospective nonrandomized comparative study conducted from June 2013 to March 2016. Patients with $\mathrm{AD}$ and abscess formation were referred to our oculo-facial clinic in a university-based hospital. All patients received systemic antibiotics and were assigned to either of treatment groups. Patients in group 1 underwent late external transcutaneous DCR (L-ExDCR) and group 2 underwent EDCR within 3 days after first visit, named VE-EDCR. Primary outcome measure was success of surgery.

Results Forty-one eyes of 41 patients with acute suppurative AD, were included from June 2013 to March 2016. Twentytwo patients underwent VE-EDCR and 19 underwent L-ExDCR. Mean age of patients was 43.41 (SD = 19.84, range 14-98) years. Mean follow-up was 14 ( $\mathrm{SD}=2.4$ ) months. Anatomic, functional, and overall success in L-ExDCR and VE-EDCR groups were $(89.5$ and $86.4 \%, p=0.99)(89.5 \%$ and $86.4 \%, p=0.99)(89.5 \%$ and $81.8 \%, p=0.66)$ respectively. Mean duration of cellulitis in VE-EDCR and L-ExDCR were $8.00(\mathrm{SD}=4.63)$ and $16.11(\mathrm{SD}=11.58)$ days, respectively $(p=$ 0.027). No remarkable adverse event was found.

Conclusions Success of very early endonasal endoscopic DCR is comparable with the traditional late external DCR. Duration of cellulitis is shorter in VE-EDCR. This therapeutic approach can be considered in patients with acute suppurative dacryocystitis.
\end{abstract}

\section{Introduction}

In acute dacryocystitis (AD), patients experience pain and swelling over the lacrimal sac and peri-orbital area secondary to acute microbial inflammation of the lacrimal sac

Farzad Pakdel

fapakdel@gmail.com

1 Oculo-Facial Surgery Department, Farabi Hospital, Eye Research Center, Tehran University of Medical Sciences, Tehran, Iran

2 Emergency Department, Farabi Hospital, Tehran University of Medical Sciences, Tehran, Iran

3 Eye Research Center, Tehran University of Medical Sciences, Tehran, Iran

4 Ophthalmology Department, Farabi Hospital, Eye Research Center, Tehran University of Medical Sciences, Tehran, Iran and surrounding tissues mostly as a result of nasolacrimal duct obstruction. It mandates antibacterial treatment for control of infection and surgical correction of lacrimal drainage obstruction. External transcutaneous DCR after complete resolution of inflammation has been the traditional surgical modality for these patients [1-6].

Endonasal endoscopic approach can provide a shorter access to the inflamed lacrimal sac through the noninflamed nasal cavity without disrupting medial canthal structures and potential skin fistula or scar formation [6]. It is assumed that early restoration of lacrimal drainage and abscess drainage in conjunction with appropriate antibiotic treatment could hasten improvement of cellulitis. Thereby, the course of antibiotic treatment, duration of patient's discomfort and number of patient's visits may be reduced. It may also be cost benefit avoiding long hospital admissions [7]. Shorter course of antibiotic therapy can reduce the drug resistance and drug associated adverse events [8]. Notably, 
also in this regard any intervention to decrease duration of antibiotic treatment and hospital admission are valuable [9].

Previous studies have shown a reasonable success of endoscopic DCR in patients with AD [7, 10, 11]. The success rate has been reported $60-94.4 \%$ [10-15] and $67-94 \%[7,10,13,16]$ of conventional late external DCR (L-ExDCR) and endonasal DCR, respectively, in patients with acute dacryocystitis. However, there are remarkable issues in the previous prior reports. Duration of presentation to operation in the prior studies was wide, from 3 to 20 days $[5,10-13]$. Obviously, such a wide time lapse in the previous studies cannot adequately support the above explained rational for an early surgical intervention. Logically, earlier surgical intervention in a reasonable time frame could result in the proposed objectives of early endonasal DCR. Thus, we defined a reasonable very early time frame for performing DCR, that is within 3 days after the first visit and taking systemic antibiotics, named as very early endoscopic DCR (VE-EDCR).

We aimed to compare the success and adverse events in the two approaches including VE-EDCR and L-ExDCR, in patients with acute suppurative dacryocystitis in a usual practice setting without selecting less complicated cases, to be representative of real lacrimal surgery practice.

\section{Methods}

This was a prospective nonrandomized comparative interventional study. Patients with acute dacryocystitis and abscess formation, referred to our clinic from June 2013 to March 2016, were included. Patients with facial nerve palsy, history of any tumor, and granulomatous diseases were excluded. History of past acute dacryocystitis, eye discharge, or epiphora were documented. Epiphora was graded with our modified Munk scoring system (Table 1). Demographic data, duration of cellulitis, systemic diseases, and the number of days taking systemic antibiotics were recorded.

All patients received systemic antibiotics and continued until clinical findings of inflammation including medial

Table 1 Modified Munk grading system for assessment of epiphora

\begin{tabular}{ll}
\hline Munk grade & \\
\hline 0 & No epiphora \\
1 & Lacrimation \\
2 & Epiphora only in wind and outdoor \\
3 & Epiphora less than twice a day \\
4 & Epiphora 2-4 times a day \\
5 & Epiphora 5-10 times a day \\
6 & Epiphora greater than ten times a day \\
7 & Continuous epiphora \\
\hline
\end{tabular}

canthal swelling, erythema, and pain were resolved. Empirical oral or intravenous antibiotics were started or continued if already started. Antibiotic was changed according to clinical response within $48 \mathrm{~h}$ considering results of culture and antibiogram results. Initial empiric intravenous antibiotic choice was cefazolin (Dana Pharma Co. Iran) $1 \mathrm{~g}$ every $6 \mathrm{~h}$ and initial oral antibiotic choice was amoxicillin/clavulanic acid $500 \mathrm{mg} / 125 \mathrm{mg}$ (Dana Pharma Co., Iran) three times a day.

This study was conducted in accordance with the declaration of Helsinki and was approved by research ethics committee. Informed consent was obtained from all patients. Patients with acute dacryocystitis and abscess were enrolled into either of the following treatment groups. Group 1 underwent L-ExDCR after receiving systemic antibiotics and when the inflammatory signs had subsided. Group 2 underwent endoscopic DCR within 3 days of starting antibiotic, named VE-EDCR regimen. In both groups, the patients were visited in a close follow-up schedule, depending on the severity of the inflammation and response to treatment, until clinical findings for cellulitis resolved. The general postoperative visit schedule was 1,3,7, 14 days, 2, 12, and 18 months. Further visits were scheduled according to the presence of active infection. In each visit, patients were asked about any discomfort, epiphora, and eye discharge. Oral antibiotic was stopped after resolution of cellulitis. Silicone tube was removed in 2 months. After tube removal irrigation and fluorescein dye disappearance tests (FDDT) were added to the postoperative evaluations.

Assessments and clinical examinations were performed by an oculo-facial fellow. Resolution of clinical findings of cellulitis, resolution of epiphora and discharge by modified Munk scale, FDDT and irrigation test were assessed and recorded in each visit. Anatomic success was defined by no reflux in irrigation test. Functional outcome was defined as absence of discharge, Munk grade 0 and negative FDDT. Successful outcome was defined by both anatomic patency and functional success components existed in the last visit at least 12 months after surgery. Functional success included absence of epiphora, discharge and abnormal FDDT and anatomic patency success included no reflux as shown in irrigation test. Primary outcome measure was successful lacrimal surgery. Secondary outcome measure was resolution of $\mathrm{AD}$ and surrounding soft tissue cellulitis.

\section{Surgical techniques}

\section{External DCR}

External DCR operations were considered to be performed either under local or general anesthesia. The site of incision was $1 \mathrm{~cm}$ nasal and inferior to medial canthus. Local 
anesthesia was performed by injection of lidocaine $2 \%$ and bupivacaine $0.5 \%$ and $1 / 100,000$ adrenaline solution to block infraorbital, infratrochlear, anterior ethmoidal nerves and local injection in the incision site. The skin was incised by a number 15 blade. Orbicularis muscle was spread over periosteum with Stevens scissors. The periosteum was incised and elevated over the nasal and lacrimal bone. Osteotomy was performed with Kerrison punch, following making a keyhole in the lacrimal bone. After tenting the lacrimal sac with a lacrimal probe 2-0, it was widely opened with " $\mathrm{H}$ " incision fashion to make a large anterior flap and small posterior part. Nasal mucosa was similarly incised as "H" fashion to make a large anterior flap and small posterior part. The posterior remnant was excised. A Bodkin silicone tube was inserted. Nasal and lacrimal mucosal flaps were stitched together with vicryl 6-0 sutures and wound was closed by nylon 6-0. After operation, patients used erythromycin ointment on the incision site for 5 days, chloramphenicol $0.5 \%$ (Sinadarou Co., Iran) and betamethasone $0.1 \%$ (Sinadarou Co., Iran) eye drops for 2 weeks, and beclomethasone nasal spray (Sinadarou Co., Iran) for 3 weeks. Skin sutures were removed in 7-10 days.

\section{Endoscopic DCR}

Operations were performed after general anesthesia. A $0^{\circ}$ or $30^{\circ}$ rigid endoscope lens (Karl Storz, Tuttlingen, Germany) was used depending on the nasal anatomy and surgeon's preference. The nasal cavity was packed with cottonoids soaked in $0.5 \%$ phenylephrine $15 \mathrm{~min}$ before the procedure. Nasal mucosa immediately anterior to the superior half of the uncinate process was incised and dissected off the bone. Frontal process of the maxilla was cut and removed with Kerrison punch. No powered instrument or laser was used. Lacrimal sac was exposed. Lacrimal sac was tented by passing a bowman 2-0 lacrimal probe. The sac was completely opened with a crescent knife. Pus drained after lacrimal sac incision was aspirated with a $10 \mathrm{ml}$ syringe and sent to bacteriology laboratory for smear, culture, and antibiogram. A silicone bicanalicular Crawford tube (FCI Co.) was inserted. Posterior lacrimal sac mucosal flap was juxtaposed with preserved nasal mucosa and tried to help this opposition by silicone tube. Intra-operative bleeding was estimated by the surgeon as less than usual, usual, or more than usual.

In both groups, betamethasone and chloramphenicol eye drop every $6 \mathrm{~h}$ for 2 weeks and beclomethasone nasal spray two puffs per day for 3 weeks were prescribed after operation. Silicone tube was removed in 2 months. In each visit the patient was assessed for clinical findings of inflammation, epiphora, and discharge. In the visits after removing the silicone tube, fluorescein dye disappearance test and irrigation test were performed. Epiphora was recorded as modified Munk scale. Fluorescein dye disappearance test was recorded $5 \mathrm{~min}$ after applying fluorescein. Any adverse perioperative event was recorded. Patients were followed for a minimum of 12 months.

Endoscopic DCR operations were performed by a single expert oculo-facial surgeon (F.P.) and external DCRs were performed by one senior oculo-facial fellow. Data were analysed by SPSS version 18. Chi-Square, Fisher exact, and Mann-Whitney tests were used for analysis.

\section{Results}

Forty-one eyes of 41 patients with suppurative AD, from June 2013 to March 2016 were included. Twenty-two patients underwent VE-EDCR and 19 patients underwent LExDCR. Demographics of the participants is shown in Table 2. Female to male ratio was 25 (60.9\%):16 (39.1\%). The mean age of the patients was 43.41 ( $\mathrm{SD}=19.84$, range 14-98) years old. Among patients, four had history of previous episodes of acute dacryocystitis in VE-EDCR group and none in L-ExDCR group $(p=0.02)$. Duration of cellulitis was $8.0(\mathrm{SD}=4.63$, range $=5.8-10.11)$ days in VE-EDCR and 16.1 $(\mathrm{SD}=15.40$, range $=8.4-23.7)$ days in L-ExDCR groups respectively $(P=0.027)$. Success rates between the two groups did not show any significant difference (Table 3). Mean follow-up time was 14.0 (SD = 2.44, range 12-21) months.

Two patients had diabetes mellitus and one patient had hypertension in the L-ExDCR group and there was one hypertensive patient in VE-EDCR group. One patient (14 years old) had cystic fibrosis in VE-EDCR group. There was a patient with severe dacryocystitis, huge lacrimal and surrounding abscess and orbital cellulitis in VE-EDCR group. All (100\%) patients in L-ExDCR and none in VEEDCR group were operated under local anesthesia. Intraoperative bleeding also was not different between the two

Table 2 Demographic data

\begin{tabular}{llll}
\hline Characteristics & VE-EDCR $^{\mathrm{a}}$ & L-ExDCR $^{\mathrm{b}}$ & $P$ value \\
\hline Age mean \pm SD (range) & $37 \pm 17$ & $51 \pm 20$ & $0.01^{\mathrm{c}}$ \\
& $(14-74)$ & $(26-98)$ & \\
Sex (M/F) & $8 / 14$ & $8 / 11$ & $0.71^{\mathrm{d}}$ \\
Laterality (L/R) & $10 / 12$ & $7 / 12$ & $0.09^{\mathrm{d}}$ \\
Presence of epiphora & $17(77 \%)$ & $12(63 \%)$ & $0.99^{\mathrm{e}}$ \\
$\begin{array}{l}\text { Duration of antibiotic } \\
\text { therapy (days) }\end{array}$ & $9 \pm 12$ & $22 \pm 16$ & $0.02^{\mathrm{c}}$ \\
\hline
\end{tabular}

${ }^{a}$ VE-EDCR: very early endoscopic DCR

${ }^{\mathrm{b}} \mathrm{L}-\mathrm{ExDCR}$ : late external DCR

${ }^{\mathrm{c}}$ Mann-Whitney test

${ }^{\mathrm{d}}$ Chi-square test

${ }^{\mathrm{e}}$ Fisher exact test 
Table 3 Anatomic, functional, and total outcome of patients in both VE-EDCR and L-ExDCR groups

\begin{tabular}{llrrrr}
\hline Characteristics & & Total & VE-EDCR $^{\mathrm{a}}$ & L-ExDCR $^{\mathrm{b}}$ & $P$ value \\
\hline Last visit epiphora $^{\mathrm{c}}$ (minimum & No & $36(87.8 \%)$ & $19(86.4 \%)$ & $17(89.5 \%)$ & 0.99 \\
12 months) & Yes & $5(12.2 \%)$ & $3(13.6 \%)$ & $2(10.5 \%)$ & \\
Last visit irrigation (minimum 12 months) & Free passage & $36(87.8 \%)$ & $19(86.4 \%)$ & $17(89.5 \%)$ & 0.99 \\
& return & $5(12.2 \%)$ & $3(13.6 \%)$ & $2(10.5 \%)$ & \\
Success & Success & $35(85.4) \%)$ & $18(81.8 \%)$ & $17(89.5 \%)$ & 0.66 \\
& failure & $6(14.6 \%)$ & $4(18.2 \%)$ & $2(10.5 \%)$ & \\
\hline
\end{tabular}

Chi-square test

${ }^{a}$ VE-EDCR: very early endonasal endoscopic dacryocystorrhinostomy

${ }^{b}$ L-ExDCR: late external transcutaneous dacryocystorrhinostomy

${ }^{c}$ Epiphora was defined as higher than grade 1 in Modified Munk scale groups $(p=0.5)$. No remarkable complication was observed in either groups.

\section{Discussion}

This study showed the trend and outcome in an occuloplastic clinic with capability for providing both treatment options including very early endonasal endscopic DCR and late external DCR protocols for the routine patients with acute dacryocystitis.

In patients with acute suppurative dacryocystitis, the conventional treatment has been systemic antiobiotic and abscess drainage through the skin. In the acute stage transcutaneous DCR approach is contraindicated in the area of acute cellulitis because of the risk of spreading infection, septicemia, bleeding, and unsatisfactory scar formation [6]. Classically, after resolution of acute inflammation, the surgeon could proceed to perform either endonasal or exteranl DCR. This late approach has potential disadvantages including risk of fistula formation, prolonged course of medical and surgical treatment, possible recurrence of infection before elective DCR surgery, risk of scar formation and anatomic disruption of medial canthal area [16]. Furthermore late approach can predispose the patient to a higher probability of systemic side effects of the antibiotics and higher risk for emergence of bacterial resistance $[8,9]$. It has been shown that decreasing duration of antibiotic therapy can decrease the two above mentioned unwanted events [8]. Early endonasal DCR can potentially address the abovementioned disadvantages of late external DCR, if performed early enough in the acute phase of dacryocystitis. Endonasal DCR has been reported as a safe procedure with comparable outcome with the traditional late transcutaneous DCR [5-7, 10, 11, 16, 17]. However, in previous studies, there has been a remarkable heterogenity of severity of cellulitis and wide time lapse of performing endo DCR after dacryocystitis from 3 to 21 days $[10,11,17]$. Obviously, there is a great difference in the amount of inflammation between the first few days and late phase in the medial canthal area. Furthermore, DCR in a later phase or unspecified time can hardly be considered an "early surgery" as some previous studies considered [7, 10, 11, 17, 18]. Addressing the primary demise of early endonasal DCR, we included patients in the first 3 days after the first visit.

VE-EDCR group included younger age compared with the traditional Ex-DCR group, $37(\mathrm{SD}=17)$ vs $51(\mathrm{SD}=$ 20) years old, respectively. We believe this can be a natural tendency of the younger people for a nonskin scar forming surgery. Also, there could be a bias for younger productive and busy individuals to be enrolled into VE-EDCR group. Further, VE-EDCR could help them to go back earlier to work.

There was a higher number of more complex patients in the VE-EDCR including patients with recurrent AD, cystic fibrosis, orbital cellulitis, and traumatic NLDO. It might be the result of a general tendency for more complex patients to be operated in an apparent more sophisticated setting. Besides, it may reflect the desire of more complex patients for an assumed newer technique. Actually, in the patients with AD, abscess and orbital cellulitis, VE-EDCR was easy, safe and quite effective to drain the lacrimal abscess and make a nasal passage for lacrimal and abscess drainage.

It has been shown that early endonasal DCR may hasten resolution of pain and swelling, in patients with acute dacryocystitis $[6,7,11,13,17]$. In the current study all patients had abscess. Abscess formation may signify more virulent pathogens, advanced infection state, and more inflammation in the medial canthal complex. Normally, these patients are potentially in higher risk of, extension of infection into orbit and they are prone to more serious complications. Therefore, they need longer duration of antibiotic treatment. Current study showed a significant difference in time of resolution of cellulitis between the two groups. This suggests that appropriate antibiotic treatment regimen and early endonasal abscess drainage can be more effective in resolution of cellulitis. Presence of abscess in all of our patients can normally prolong the resolution time. 
More complex cases in VE-EDCR group should not be overlooked.

The success rate of VE-EDCR and L-ExDCR in our study was $81.8 \%$ and $89.5 \%$, respectively. The surgical success results in the treatment arms are in concordance with two recent comparative studies $[11,13]$. Joshi et al. [13] in a retrospective study, compared the success rate among 57 patients with acute dacryocystitis and found no significant difference in success between early endoscopic DCR $(82.1 \%, n=28)$ and late external DCR $(89.7 \%, n=$ 26) groups. The success rate among VE-EDCR patients in our study $(81.8 \%)$ is nearly comparable with those reported by Wu et al. (87\%) [10], Madge et al. (94\%) [7], and Duggal et al. (72\%) [19].

In another study done by $\mathrm{Wu}$ et al. [10] the success rate of delayed Ext-DCR (1-2 weeks) in patients with acute dacryocystitis was $60 \%$ in Ext-DCR group compared with 90\% in the early En-DCR group with silicone tube and a soft probe. The lower success in the earlier studies may have been explained by episodes of dacryocystitis [13] and inflammation of the median canthal area [17]. In our study four patients had a history of previous acute dacryocystitis (four in the VEEDCR group). One of these patients failed the surgery.

The average duration of cellulitis in the patients in the VE-EDCR group (8 days) was almost half of those in LExDCR group (16 days). Li et al. [11] reported 32 patients with acute dacryocystitis and lacrimal sac abscess that underwert primary En-DCR within 2 weeks or secondary En-DCR after 30 days of medical treatment and percutaneous abscess drainage. They found faster resolution of symptoms in the primary endoscopic DCR group (13.8 days) compared with the control group (31.7 days). The success rate (anatomical and functional) in both groups of their patients was $87.5 \%$ (14 of 16 cases).

Interestingly, early nonendoscopic endonasal may have a similar result to the early endonasal endoscopic surgery. Jain et al. compared the outcome of early nonendoscopic endonasal DCR with mitomycin C within 2 weeks of presentation, and Ext-DCR performed 3 weeks after resolution of acute dacryocystitis. They found similar success rate $(86 \%)$ in both groups [17].

Some argue that delayed surgery carries the risk of recurrence in up to $14 \%$ of patients with acute dacryocystitis $[17,20]$. We did not find recurrence in $\mathrm{AD}$ in any of our patients.

The number of participants of the current study is near or more than other similar prospective comparative $(n=32)$ [11] and retrospective comparative studies $(n=46-57)$ $[13,17,18]$ on acute dacryocystitis.

Interpreting the results, it is remarkable to note that, the sample of patients in this study included real oculo-facial clinic of patients with $\mathrm{AD}$ that comprise traumatic, recurrent subjects and those with systemic morbidities. Hence, it can be more representative of the usual $\mathrm{AD}$ patients and higher potential for generalizability of the current study results.

To the best of our knowledge, this is the first comparative study on very early endonasal endoscopic DCR in patients with acute dacryocystitis and abscess formation.

This study showed that VE-EDCR is at least comparable with a late external DCR in terms of success and complications in a routine group of patients admitted to oculoplastic clinic. Further this approach resulted in shortening duration of cellulitis and time of antibiotic therapy.

All patients in the VE-EDCR group were operated under general anesthesia. Actually, we do operate patients with endonasal endoscopic approach under either local or general anesthesia depending on the general condition of the patient and patient preference. However, in patients with cellulitis general anesthesia is preferred. Intra-operative bleeding between the two groups were not significantly different. We hypothesized that, by endonasal endoscopic approach to the lacrimal sac in the early stage of cellulitis, the surgery is directed through the noninflammed area. This anatomical issue can be at least one of the factors for the observed similar intraoperative bleeding in the early and late surgery groups.

Interestingly, it was found that about $30 \%$ of patients did not report any history of epiphora before developing acute dacryocystitis. We assumed a few reasons to explain the absence of epiphora in a subset of patients. First, acute dacryocystitis can be the first manifestation of a recent onset nasolacrimal duct obstruction. Second, patients with dry eye and tear hyposecretion may not complain of epiphora in the presence of nasolacrimal duct obstruction. Third, theoretically acute dacryocystitis can occur as a result of a recent stagnation of tear flow through lacrimal sac secondary to dacryoliths. Reported demographics of patients in a study on patients with acute dacryocystitis showed that $21.5 \%$ of patients with acute dacryocystitis had no preexisting nasolacrimal duct obstruction [11]. This is a close percentage to that in our study and supports this issue. This phenomenon has been rarely addressed in the literature and needs to be clarified by future studies. Absence of epiphora in some of the patients with AD could not impact validity of outcome measure. Because success outcome has be defined on the basis of both anatomic and fuctional status of lacrimal draininage and not on epiphora per se.

Limitations in the current study include undetailed detection and control of confounders such as pathogenic organisms, antibiotic regimens between the study groups, aestheic evaluation, and limited sample size.

In conclusion, very early EDCR in acute suppurative dacryocystitis could both hasten resolution of cellulitis and abscess and resolve the nasolacrimal duct obstruction with a low morbidity. VE-EDCR can be regarded as an alternative management option in patients with acute suppurative dacryocystitis. 


\section{Summary}

\section{What was known before}

- For a long time, systemic antibiotics and late external dacryocystorhinostomy (L-ExDCR) have been the standard of care in patients with acute dacryocystitis (AD). Further, in those who develop abscess, transcutaneous drainage could be added to the treatment plan.

- Recently, endoscopic dacryocystorhinostomy (EDCR) in the inflammatory stage of $\mathrm{AD}$ has been introduced in acute dacryocystitis. However, the time lag in previous reports has been quite wide from 5 days to 3 weeks.

\section{What this study adds}

- Very early endonasal endoscopic DCR within 3 days after presentation, may improve patient care in acute dacryocystitis.

- It can decrease the course of antibiotic therapy.

- In addition, it may decrease drug related adverse events and emergence of antibiotic resistant bacteria.

- Finally, number of visits can be decreased.

\section{Compliance with ethical standards}

Conflict of interest The authors declare that they have no conflict of interest.

Publisher's note Springer Nature remains neutral with regard to jurisdictional claims in published maps and institutional affiliations.

\section{References}

1. Roithmann R, Burman T, Wormald PJ. Endoscopic dacryocystorhinostomy. Braz J Otorhinolaryngol. 2012;78:113-21.

2. Massaro BM, Gonnering RS, Harris GJ. Endonasal laser dacryocystorhinostomy. A new approach to nasolacrimal duct obstruction. Arch Ophthalmol 1990;108:1172-6.

3. McDonogh M, Meiring JH. Endoscopic transnasal dacryocystorhinostomy. J Laryngol Otol. 1989;103:585-7.
4. Wormald PJ, Kew J, Van Hasselt A. Intranasal anatomy of the nasolacrimal sac in endoscopic dacryocystorhinostomy. Otolaryngol Head Neck Surg. 2000;123:307-10.

5. Tsirbas A, Wormald PJ. Mechanical endonasal dacryocystorhinostomy with mucosal flaps. Otolaryngol Clin North Am. 2006;39:1019-36.

6. Lee TS, Woog JJ. Endonasal dacryocystorhinostomy in the primary treatment of acute dacryocystitis with abscess formation. Ophthalmic Plast Reconstr Surg. 2001;17:180-3.

7. Madge SN, Chan W, Malhotra R, et al. Endoscopic dacryocystorhinostomy in acute dacryocystitis: a multicenter case series. Orbit 2011;30:1-6.

8. Bell BG, Schellevis F, Stobberingh E, et al. A systematic review and meta-analysis of the effects of antibiotic consumption on antibiotic resistance. BMC Infect Dis. 2014;9:13.

9. Davey P, Marwick CA, Scott CL, et al. Interventions to improve antibiotic prescribing practices for hospital inpatients. Cochrane Database Syst Rev. 2017;2:CD003543.

10. Wu W, Yan W, MacCallum JK, et al. Primary treatment of acute dacryocystitis by endoscopic dacryocystorhinostomy with silicone intubation guided by a soft probe. Ophthalmology. 2009; 116:116-22.

11. Li EY, Wong ES, Wong AC, et al. Primary vs secondary endoscopic dacryocystorhinostomy for acute dacryocystitis with lacrimal sac abscess formation: a randomized clinical trial,". JAMA Ophthalmol. 2017;135:1361-6.

12. Saha R, Sinha A, Phukan JP. Endoscopic versus external approach dacryocystorhinostomy: a comparative analysis. Niger Med J. 2013;54:165-9.

13. Joshi RS, Deshpande AS. Success rate of conventional dacryocystorhinostomy in post-acute dacryocystitis compared to endonasal dacryocystorhinostomy in acute dacryocystitis. J Ophthalmic Vis Res. 2017;12:290-5.

14. Rabina G, Golan S, Neudorfer M, et al. External dacryocystorhinostomy: characteristics and surgical outcomes in patients with and without previous dacryocystitis. J Ophthalmol 2013;2013:287524.

15. Amadi AJ. Endoscopic DCR vs external DCR: What's best in the acute setting? J Ophthalmic Vis Res. 2017;12:251-3.

16. Morgan S, Austin M, Whittet $\mathrm{H}$. The treatment of acute dacryocystitis using laser assisted endonasal dacryocystorhinostomy. $\mathrm{Br}$ J Ophthalmol. 2004;88:139-41.

17. Jain S, Ganguly A, Singh S, et al. Primary nonendoscopic endonasal versus delayed external dacryocystorhinostomy in acute dacryocystitis. Ophthalmic Plast Reconstr Surg. 2017;33:285-8.

18. Naik SM, Appaji MK, Ravishankara S, et al. Acute dacryocystitis with abscess: endonasal dacryocystorhinostomy, the primary treatment of choice. Clin Rhinol. 2012;5:107-13.

19. Duggal P, Mahindroo NK, Chauhan A. Primary endoscopic dacryocystorhinostomy as treatment for acute dacryocystitis with abscess formation. Am J Otolaryngol. 2008;29:177-9.

20. Ali MJ, Joshi SD, Naik MN, et al. Clinical profile and management outcome of acute dacryocystitis: two decades of experience in a tertiary eye care center. Semin Ophthalmol 2015;30:118-23. 